\title{
Tasas Regionales de hospitalización y mortalidad por asma infantil en Chile
}

\author{
Regional asthma hospitalization rates and global asthma mortality rate \\ in Chilean children
}

\section{Ana María Herrera Gana ${ }^{\mathrm{a}, \mathrm{b}}$, Gabriel Cavada Chacón ${ }^{\mathrm{c}}$}

aClínica Santa María, Santiago, Chile

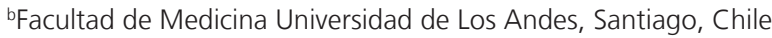

Universidad Finis Terrae, Santiago, Chile

Recibido: 30 de octubre de 2019; Aceptado: 20 de abril de 2020

¿Qué se sabe del tema que trata este estudio?

En el último tiempo se ha observado un aumento significativo de la tasa global de hospitalización por asma en niños desde 3,8 a 7,8 por 10.000 habitantes.

\section{¿Qué aporta este estudio a lo ya conocido?}

Existen algunas diferencias regionales en las tasas de hospitalización por asma en niños, siendo la $\mathrm{V}$ Región la que presenta la tasa más alta. La tasa global de mortalidad por asma en niños es de 0,37 por 100.000 habitantes.

\section{Resumen}

En los últimos años se ha observado un aumento significativo de la tasa de hospitalización por asma en niños. En la actualidad se desconocen las tasas regionales y la tasa de mortalidad. Objetivo: Conocer las tasas regionales de hospitalización por asma en niños y determinar la mortalidad en este grupo etario. Pacientes y Método: Se calculó la tasa de hospitalización por asma en las 15 regiones del país existentes al momento de realizar el estudio, entre el año 2008 y 2014, en base al número de egresos hospitalarios por región y de las poblaciones expuestas al riesgo de hospitalización. La tasa de mortalidad se obtuvo calculando el cociente entre el número de defunciones por asma en niños de 5 a 15 años y la población expuesta. Resultados: La tasa más alta de hospitalización la presentó la $\mathrm{V}$ región con 7,6 por 10.000 habitantes. La tasa media de hospitalización en las distintas regiones fue similar a la de la Región Metropolitana, con la excepción de 4 regiones. La tasa global de mortalidad por asma en niños de 5 a 15 años para el periodo analizado es de 0,37 por 100.000 habitantes. Conclusiones: La mayoría de las regiones del país presentan tasas de hospitalización similares a la Región Metropolitana. La V Región presenta la tasa más alta de hospitalización. La tasa global de mortalidad por asma en niños de 5 a 15 años es de 0,37 por 100.000 habitantes.
Palabras clave:

Asma;

niños;

hospitalización;

mortalidad 


\begin{abstract}
In recent years, there has been a significant increase in asthma hospitalization rates in children, however, regional and mortality rates are yet unknown. Objective: To determine regional asthma hospitalization rates in children and the global mortality rate in this age group. Patients and Method: We determined asthma hospitalization rates in the 15 regions of the country existing at the time of the study, between 2008 and 2014, based on the number of hospital discharges in each region and the population at risk of hospitalization. The mortality rate was obtained using the ratio between deaths due to asthma in children aged 5 to 15 and the exposed population. Results: the $5^{\text {th }}$ region presented the highest hospitalization rate (7.6 per 10,000 inhabitants). Except for 4 regions, the median hospitalization rates of the different regions were similar to those found in the Metropolitan Region. The overall mortality rate due to asthma in 5 to 15 -year-old children was 0.37 per 100,000 inhabitants in the analyzed period. Conclusions: most regions of the country have similar hospitalization rates to the Metropolitan Region and the $5^{\text {th }}$ region presents the highest hospitalization rate due to asthma. The global mortality rate in children between 5 and 15 years old is 0.37 per 100,000 inhabitants.
\end{abstract}

\section{Keywords:}

Asthma;

children;

hospitalization;

mortality

\section{Introducción}

El asma bronquial es una enfermedad altamente prevalente a nivel mundial. En Latinoamérica la prevalencia de asma en niños varía entre un 2,6\% y un $33,1 \%$, siendo mayor al $15 \%$ en más de la mitad de los países de la región ${ }^{1}$. Utilizando cuestionarios estandarizados The International Study of Asthma and Allergies in Childhood (ISAAC, estudio internacional de asma y alergias en la infancia) determinó que la prevalencia de asma en Chile en el grupo de 6 a 7 años era de 17,9\% y en el grupo de 13 a 14 años de $15,5 \%{ }^{2}$. Las hospitalizaciones por asma bronquial representan un resultado adverso serio en los pacientes con "asma severo" y "asma de difícil control"3. En un estudio publicado en Chile se observó un aumento significativo de la tasa de hospitalización por asma en niños de 5 a 15 años desde el año 2001 en que la tasa era de 3,8 por 10.000 habitantes a 7,8 por 10.000 habitantes el año $2014^{4}$. En el período tiempo estudiado se observó que el incremento más significativo de las tasas de hospitalización ocurrió en niños de 5 a 7 años, sin encontrarse diferencias significativas por sexo. La letalidad reportada fue de $0,02 \%{ }^{4}$.

El objetivo del presente estudio es estimar las tasas regionales de hospitalización por asma en niños de $5 \mathrm{a}$ 15 años, evaluar si existen diferencias entre ellas y determinar la mortalidad por asma en este grupo etario. Estos datos nos permitirán identificar a las regiones que tienen las tasas más altas de hospitalización por asma y de esta manera diseñar estrategias para reducirlas.

\section{Pacientes y Método}

\section{Diseño}

Estudio de tipo observacional, descriptivo de la evolución de las tasas de hospitalización por asma, desde el año 2008 al 2014, en niños cuyas edades fluctua- ron entre los 5 y los 15 años en las 15 regiones de Chile, existentes al momento de realizar el estudio. La evolución de las tasas se analizó en forma global por región.

\section{Obtención de datos}

Los datos fueron extraídos de los registros de egresos hospitalarios proporcionados por el Departamento de Estadística e Información de Salud del Ministerio de Salud de Chile $(\mathrm{DEIS})^{5}$. Este registro incluye todos los egresos hospitalarios a nivel nacional incluyendo tanto los del sector público como del privado. Se seleccionó la información correspondiente a los egresos hospitalarios por asma (códigos CIE10: J450, J451, J458, J459 y J46), además de la edad simple, sexo, días de hospitalización, fecha de hospitalización y la condición de egreso. Los tamaños de las poblaciones expuestas al riesgo de hospitalización se obtuvieron de las proyecciones de población proporcionadas por el Instituto Nacional de Estadística de Chile (INE) ${ }^{6}$.

\section{Análisis de datos}

La evolución de las tasas de hospitalización se analizó mediante un modelo lineal autoregresivo de primer orden (Prais-Winsten). Las comparaciones entre rangos etarios y regiones geográficas se realizaron mediante un análisis de regresión de Poisson, donde la medida de comparación fue la razón de tasas de incidencia (IRR). Como la Región Metropolitana es la con mayor número de habitantes y la más estable desde el punto de vista poblacional, se decidió considerar a ésta como la región de referencia para comparar las tasas de hospitalización por asma en las otras regiones del país. La tasa de mortalidad se obtuvo calculando el cociente entre el número de defunciones por asma en niños de 5 a 15 años y la población expuesta, obtenida de las proyecciones de población proporcionadas por el INE6. Las variables continuas se describieron mediante, mediana 
y rango intercuartílico (RIC), mientras que las variables cualitativas mediante frecuencias y proporciones. Los intervalos de confianza fueron de nivel $95 \%$ y se utilizó una significación del 5\%. Los datos fueron procesados en el programa estadístico STATA 14.0.

\section{Resultados}

Durante el período estudiado hubo un total de 29.821 egresos hospitalarios por asma, de los cuales $10.847(36,4 \%)$ correspondieron a niños de 5 a 15 años. La mediana de edad fue de 8 (RIC 6-10) y no varió en el período de tiempo estudiado $(\mathrm{p}=0,868)$. El $78 \%$ del total de niños hospitalizados fueron menores de 10 años. Esta mayor proporción de hospitalización en niños menores se presenta en todas las regiones del país. Al modelar el número de egresos hospitalarios por asma explicado por el rango etario de 11 a 15 años versus de 5 a 10 años, ajustado por región, se observa que los niños de 5 a 10 años tienen 3 veces más riesgo de hospitalización que el grupo de mayor edad $($ IRR $=3,11$ IC95\% 2,98-3,26 p < 0,001). Del total de egresos hospitalarios $6.251(57,6 \%)$ correspondieron a pacientes de sexo masculino, mientras que 4.596 $(42,4 \%)$ a sexo femenino. Al analizar las hospitalizaciones por región no hubo diferencias significativas por sexo. La mediana de días de hospitalización fue de 3 (RIC 1-5) para ambos sexos. Esta mediana se observó consistentemente en todas las regiones excepto en la IV región, en la que la mediana fue de 4 días, siendo esta diferencia significativa $(\mathrm{p}<0,0001)$. La mediana de días de hospitalización no varió en el período de tiempo analizado. La tasa media global de hospitalización en los 7 años analizados fue de 4,5 por 10.000 habitantes (IC 95\%, 4,04-4,92) manteniéndose estable durante el período de tiempo analizado $(\mathrm{p}=0,404)$. La mayor tasa media de hospitalización la presenta la $\mathrm{V}$ región con 7,6 por 10.000 habitantes seguida por la IV y IX regiones con 6,0 y 5,9 por 10.000 habitantes respectivamente (tabla 1). Por otro lado las tasas medias de hospitalización más bajas corresponden a la VII región con 1,7 por 10.000 habitantes, seguida por la XII y III regiones con 1,9 y 2,3 por 10.000 habitantes respectivamente (tabla 1 ).

Al comparar las tasas medias de hospitalización de cada región con las de la Región Metropolitana (RM), observamos que las regiones III, VII y XII presentan tasas significativamente menores que ésta. Por otro lado, la $\mathrm{V}$ región presenta una tasa de hospitalización significativamente mayor que la de la RM. Las tasas medias de hospitalización en las restantes regiones no difieren significativamente de las de la RM (tabla 1).

$\mathrm{Al}$ analizar la evolución de las tasas de hospitalización por regiones durante el período estudiado se observó que en todas las regiones estas tasas se mantienen constantes, con excepción de la I región que presenta una disminución de 0,7 hospitalizaciones por 10.000 habitantes por año $(\mathrm{p}=0,019)$ y la VIII región que presenta un aumento de 0,2 hospitalizaciones por 10.000 habitantes por año $(\mathrm{p}=0,016)$.

Tabla 1. Tasa media de hospitalización por asma por región y comparación de las tasas medias regionales con la tasa media de la Región Metropolitana

\begin{tabular}{ccc}
\hline Región & $\begin{array}{c}\text { Tasa media de hospitalización por región } \\
\text { por } \begin{array}{c}10.000 \text { habitantes } \\
(2008-2014)\end{array}\end{array}$ & $\begin{array}{c}\text { Comparación de las tasas medias regionales con la tasa } \\
\text { media de la Región Metropolitana (XIII) } \\
\text { Valor p }\end{array}$ \\
\hline I & 3,9 & 0,351 \\
II & 5,4 & 0,412 \\
III & 2,3 & $0,005^{*}$ \\
IV & 6,0 & 0,125 \\
V & 7,6 & $<0,001^{*}$ \\
VI & 3,4 & 0,131 \\
VII & 1,7 & $0,001^{*}$ \\
VIII & 5,6 & 0,269 \\
IX & 5,9 & 0,167 \\
X & 4,8 & 0,906 \\
XI & 5,5 & 0,328 \\
XII & 1,9 & 0,001 * \\
XIV & 4,5 & 0,853 \\
XV & 4,0 & 0,408 \\
XIII & 4,7 & Referencia (Región Metropolitana)
\end{tabular}

*Diferencia estadísticamente significativa. 
La tasa global de mortalidad por asma en niños de 5 a 15 años para el periodo analizado fue de 0,37 por 100.000 habitantes, siendo la mayoría de los pacientes fallecidos (5/7) mayores de 10 años.

\section{Discusión}

Las hospitalizaciones por asma reflejan el estado máximo de morbilidad y son un indicador de mal control de la enfermedad y en menor medida de la severidad intrínseca de la misma. En Chile hemos visto que las tasas de hospitalización por asma en niños se han duplicado en los últimos años, constituyendo un importante problema de salud pública que debemos abordar como país ${ }^{4}$. Del total de pacientes asmáticos hospitalizados en Chile durante el período analizado, $40 \%$ corresponde a niños de 5 a 15 años y dentro de este grupo casi un $80 \%$ son menores de 10 años. La mayor proporción de pacientes de menor edad hospitalizados por asma se observa consistentemente en todas las regiones de nuestro país. La mayor proporción de hospitalizaciones en pacientes asmáticos de menor edad también ha sido reportada en otros países como Estados Unidos y España ${ }^{7,8}$. Es posible que el mayor número de hospitalizaciones en este grupo etario sea debido a que en edades más tempranas puede haber un subdiagnóstico de la enfermedad y por tanto falta de tratamiento adecuado. Otra posibilidad es la mayor exposición a las infecciones virales a las que están sometidos los niños más pequeños y que como se sabe es el principal gatillo para las exacerbaciones de asma.

En general las tasas de hospitalización en las distintas regiones son similares a las de la región metropolitana. Pese a lo anterior se observaron diferencias significativas en 4 regiones (III, V, VII, XII). En la literatura internacional también se han descrito diferencias importantes en las tasas de hospitalización por asma en niños de países vecinos, con características climáticas y de desarrollo económico similar como Finlandia y Suecia. Es así como en Finlandia se reportan tasas de hospitalización de 6,8 por 10.000 habitantes el año 2014 mientras que en Suecia la tasa en el mismo período fue de 13,6 por 10.000 habitantes ${ }^{9}$. Así mismo se han descrito diferencias regionales en las tasas de hospitalización por asma infantil dentro de un mismo país. Tal es el caso de Estados Unidos donde se observó que las tasas de hospitalización eran más bajas en la región oeste del país, seguido por las regiones nororiente, central y sur ${ }^{10}$. La causa de las diferencias regionales en las tasas de hospitalización por asma probablemente es de origen multifactorial. Uno de estos factores pudiera ser el diferente grado de exposición a factores ambientales como virus, alérgenos y contaminantes ambientales intra y extra domiciliarios. Al respecto la contaminación ambiental pudiera jugar un rol importante en la mayor tasa de hospitalización por asma que presenta la $\mathrm{V}$ región de nuestro país, donde se ubican varias centrales termoeléctricas y refinerías de petróleo y donde se han reportado varias alertas ambientales en el último tiempo. Hay múltiples estudios en la literatura internacional que demuestran una clara asociación entre el nivel de contaminación ambiental de un lugar determinado y el número de hospitalizaciones por asma. Los contaminantes que pueden causar problemas en los pacientes asmáticos son el material particulado 2.5 (PM2.5), material particulado 10 (PM10), ozono (O3), dióxido de sulfuro (SO2) y dióxido de nitrógeno (NO2). En un metanálisis publicado por Zheng el 2015 y que incluyó 87 estudios se demostró que la exposición a corto plazo a todos estos contaminantes aumentaba el riesgo de consultas a servicios de urgencia y hospitalizaciones por asma especialmente en niños y adultos mayores ${ }^{11}$. Otro estudio realizado en Taiwan, durante un año calendario, evaluó si existía correlación entre los niveles de contaminación ambiental en las distintas regiones del país con las hospitalizaciones por asma en el mismo período. Se observó que esta asociación era más intensa en aquellas regiones con niveles más altos de contaminación mientras, que era débil en las regiones con bajos niveles ${ }^{12}$. Las refinerías de petróleo son fuente de muchos contaminantes especialmente PM 10, SO2 y NO2. En un estudio de corte transversal realizado en Quebec, que es una ciudad en la cual existen refinerías de petróleo, se observó que había una clara asociación entre los niveles de $\mathrm{SO} 2$ y el diagnóstico de asma en aquellos niños que vivían en las cercanías de estas refinerías, así como también con el número de niños con asma no controlado ${ }^{13}$.

Otros factores que pudieran influir en las variaciones regionales de las tasas de hospitalización son el distinto nivel de severidad de los pacientes, distinto grado de control de la enfermedad y un mejor o peor manejo ambulatorio ${ }^{14-16}$. Con respecto a la influencia que un manejo ambulatorio deficiente pudiera tener sobre las hospitalizaciones por asma, vemos que en un estudio descriptivo, prospectivo multicéntrico realizado en niños chilenos hospitalizados por crisis de asma se observó que casi $50 \%$ de estos pacientes no tenían diagnóstico establecido de asma bronquial al momento de la hospitalización y por tanto estaban sin recibir tratamiento de mantención. Por otro lado, los pacientes que sí tenían diagnóstico establecido de asma bronquial presentaban mala adherencia al tratamiento de mantención ${ }^{17}$. El uso del tratamiento de mantención es fundamental para mantener al asma bajo control y evitar así las crisis, por tanto este es un factor muy importante a tener en consideración en nuestro país si queremos reducir en parte las hospitalizaciones por asma en niños. 
La tasa de mortalidad por asma para el periodo analizado es baja, comparable con la que presentan países desarrollados. Llama la atención que del total de niños fallecidos por asma en nuestro país, la gran mayoría de ellos era mayor de 10 años. Esta observación contrasta con el hecho que este grupo etario es el que menos se hospitaliza, con lo cual se podría elucubrar que estos pacientes probablemente no están en control médico, subvaloran sus síntomas y por tanto están expuestos a mayor riesgo de morir a causa de esta enfermedad. Ciertamente la adolescencia es una etapa complicada de la vida en la cual los pacientes están desarrollando su autonomía y al mismo tiempo se alejan de los padres y sus cuidados. Por tanto, sería importante desarrollar en nuestro país programas de seguimiento especiales para este grupo vulnerable de pacientes con asma.

Nuestro estudio tiene la fortaleza de mostrar por primera vez en Chile las variaciones regionales de las tasas de hospitalización por asma en niños y al mismo tiempo la mortalidad global por asma infantil. La principal debilidad es la calidad de los registros disponibles, ya que no contamos con datos detallados de las hospitalizaciones, como severidad del asma, presencia de comorbilidades, adherencia al tratamiento de mantención o tipo de terapia recibida por cada paciente.

Nos parece de suma importancia poder dilucidar los principales factores de riesgo presentes en las re- giones que presentan las tasas de hospitalización más altas, de manera de poder implementar las medidas necesarias tendientes a reducirlas.

\section{Responsabilidades Éticas}

Protección de personas y animales: Los autores declaran que los procedimientos seguidos se conformaron a las normas éticas del comité de experimentación humana responsable y de acuerdo con la Asociación Médica Mundial y la Declaración de Helsinki.

Confidencialidad de los datos: Los autores declaran que han seguido los protocolos de su centro de trabajo sobre la publicación de datos de pacientes.

Derecho a la Privacidad y Consentimiento Informado: Los autores declaran que la información ha sido obtenida de datos previos en forma anonimizada, por lo cual el Comité de Ética de Investigación en uso de sus facultades, ha eximido de la obtención de un consentimiento informado, lo cual consta en el acta respectiva.

\section{Conflicto de intereses}

Los autores declaran no tener conflicto de intereses.

\section{Referencias}

1. Forno E, Gogna M, Cepeda A, et al. Asthma in Latin America. Thorax 2015;70:898-905.

2. Mallol J, Aguirre V, Aguliar P, et al. Cambios en la prevalencia del asma en escolares chilenos entre 1994 y 2002. Rev Med Chile 2007;135:580-6.

3. Fan Chung K, Wenzel SE, Brozek JL, et al. International ERS/ATS guidelines on definition, evaluation and treatment of severe asthma. Eur Respir J 2014;43:34373.

4. Herrera AM, Cavada G, Mañalich J. Hospitalizaciones por Asma Infantil en Chile: 2001-2014. Rev Chil Pediatr. 2017;88(5):602-7.

5. https://reportesdeis.minsal.cl/ egresoshospitalarios/menu_publica_ nueva/menu_publica_nueva.htm (accesado en junio 2019).

6. http://www.ine.cl/estadisticas/ demograficas-y-vitales (accesado en junio 2019).

7. Hasegawa K, Tsugawa Y, Brown D, Camargo C. Childhood asthma hospitalizations in the United States,
2000-2009. J Pediatr 2013;163:1127-33.

8. De Miguel-Díez J, Jiménez-García R, Hernández-Barrera V, López de Andrés A, Villa-Asensi J, Plaza V, et al. National trends in hospital admissions for asthma exacerbations among pediatric and Young adult population in Spain (2002-2010). Respir Med 2014;108:983-91.

9. Kivistö JE, Protudjer JLP, Karjalainen J, Bergström A, Korppi M. Trends in paediatric asthma hospitalisationsdifferences between neighbouring countries. Thorax 2018;73(2):185-7.

10. Gerhardsson de Verdier M, Gustafson P, McCrae C, Edsbäcker S, Johnston N. Seasonal and geographic variation in the incidence of asthma exacerbations in the United States. J Asthma 2017; 54(8):81824.

11. Zheng XY, Ding H, Jiang LN, et al. Association between Air Pollutants and Asthma Emergency Room Visits and Hospital Admissions in Time Series Studies: A Systematic Review and MetaAnalysis.

12. Kuo CY, Chan CK, Wu CY, Phan DV, Chan CL. The Short-Term Effects of Ambient Air Pollutants on Childhood
Asthma Hospitalization in Taiwan: A National Study. Int J Environ Res Public Health. 2019;16(2):203.

13. Deger L, Plante C, Jacques L, et al. Active and uncontrolled asthma among children exposed to air stack emissions of sulphur dioxide from petroleum refineries in Montreal, Quebec: A cross-sectional study. Can Respir J. 2012;19: 97-102.

14. Homa DM, Mannino DM, Redd SC. Regional Differences in Hospitalizations for Asthma in the United States, 19881996. J Asthma 2002;39:449-55.

15. Goodman DC, Stukel TA, Chang $\mathrm{CH}$. Trends in Pediatric Asthma Hospitalization Rates: Regional and Socioeconomic Differences. Pediatrics 1998;101:208-13.

16. Roy SR, McGinty EE, Hayes SC, Lei Zhang L. Regional and racial disparities in asthma hospitalizations in Mississippi. J Allergy Clin Immunol 2010;125:636-42.

17. Herrera AM, Brand P, Cavada G, Koppmann A, Rivas M, Mackenney J, et al. Hospitalizations for asthma exacerbation in Chilean children: A multicenter observational study. Allergol Immunopathol (Madr). 2018;46:533-8. 